\title{
ULTRAVIOLET AND OPTICAL SPECTRA OF CENTRAL STARS OF HALO PLANETARY NEBULAE
}

\author{
M. PEÑA and S. TORRES-PEIMBERT \\ Instituto de Astronomía, Universidad Nacional Autónoma de México, Apdo. Postal 70264, \\ 04510 Méx. D.F., México \\ and \\ M. T. RUIZ \\ Departamento de Astronomía, Universidad de Chile, Casilla 36-D, Santiago, Chile
}

ABSTRACT. Optical and UV spectrophotometric data are analyzed for the central stars of the known Population II planetary nebulae. From this, we derive visual magnitudes, spectral classification, color temperatures, luminosities and masses of the objects. It is found that all the stars show absorption type spectrum and most of them have normal $\mathrm{H}$ and He photospheric abundances, the only possible exceptions are M 2-29 and GJJC-1 which seem to be H-deficient stars.

The visual magnitudes, derived from UV stellar continuum, are in good agreement with magnitudes derived from photometric optical data, except for NGC 2242 for which we found a value $V=17.1$ mag instead of the 15.02 reported by Maehara et al (1987), this implies a distance 2 kpc larger than that calculated by Torres-Peimbert et al. (1990). High signal-to-noise photometric observations of the central star are required to confirm these results.

The color temperatures derived for these objects range from less than $40,000 \mathrm{~K}$ to more than $80,000 \mathrm{~K}$ and, in general, they are consistent with effective temperatures calculated by other means. We found that the central star of NGC 4361 shows a UV continuum steeper than expected from the effective temperature determined from modelling of $\mathrm{H}$ and $\mathrm{He}$ absorption lines. From comparison with theoretical evolutionary tracks for nuclei of PN we found that the stellar masses of these objects spread over a na- rrow range from 0.55 to $0.58 \mathrm{M}(\odot)$. The average mass is lower than the average masses derived for large samples of disk and bulge planetary nebulae.

References

Maehara, H., Okamura, S, Noguchi, T., He, X-T., Liu, J., Huang, Y.W. and Feng, X.C. (1987) A\&A, 178, 221

Torres-Peimbert, S., Peimbert, M. and Peña, M. (1990) A\&A, 233, 540 